\title{
Le bonheur est-il dans la pilule?
}

\section{Julia Rippstein}

Rédactrice print online

Pourquoi prend-on des médicaments, pilules et autres vitamines? Explorant la question, le journaliste suisse Arnaud Robert et le photographe Paolo Woods ont sillonné le globe pendant cinq ans. Le résultat est à voir au travers de l'exposition HAPPY PILLS jusqu'au 16 janvier 2022 à La Ferme des Tilleuls de Renens.

Nous avons tous avalé un jour ou l'autre un médicament - du moins dans les pays «riches». Derrière la prise de comprimés, une douleur, une blessure, un dysfonctionnement, un mal-être. Ces produits miracle sont censés nous aider à aller mieux. L'exposition HAPPY PILLS se penche sur ces "pilules du bonheur»,

«La médecine crée tout un tas de diagnostics qui définissent des troubles auxquels

les médicaments donnent une réponse."

allant du Viagra à la Ritaline en passant par les anxiolytiques, et les raisons qui nous poussent à en prendre. Curieux de comprendre et de questionner notre rapport à ces petites gélules, le journaliste vaudois Arnaud
Robert et le photographe canado-néerlandais Paolo Woods ont parcouru les quatre coins du monde à la rencontre de six consommatrices et consommateurs de médicaments en quête de bonheur. HAPPY PILLS plonge le visiteur dans une réelle enquête journalistique composée de photographies, de témoignages poignants et de séquences filmées. Le Bulletin des médecins suisses s'est rendu sur place.

\section{Marchands de rêves?}

La genèse du projet HAPPY PILLS remonte à 2014 en Haïti, où Paolo Woods et Arnaud Robert collaborent alors sur deux ouvrages. Leur attention est retenue par les marchands de médicaments qui arpentent les rues

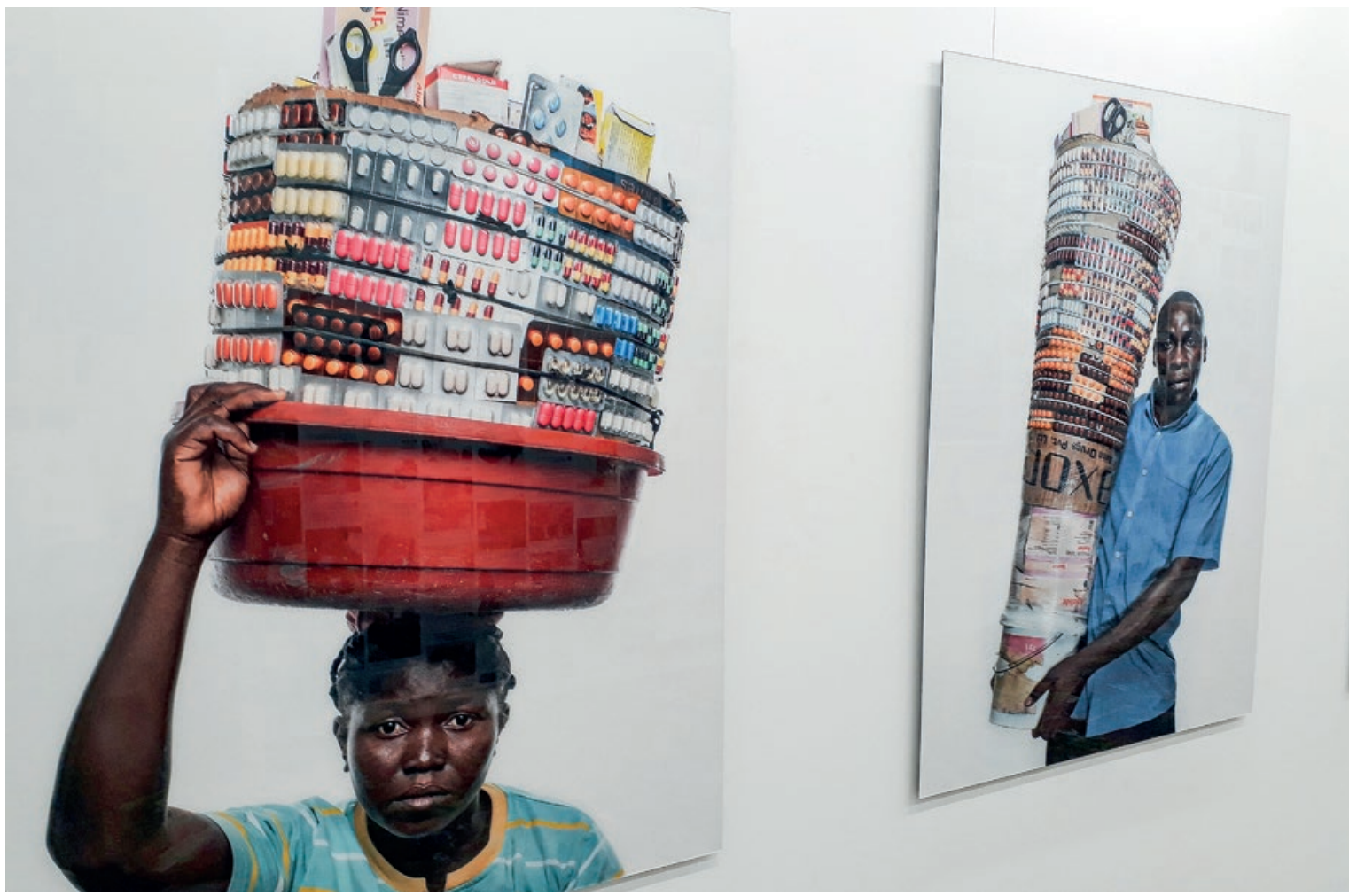

Les vendeurs de médicaments en Haïti ont marqué le début du projet HAPPY PILLS. 


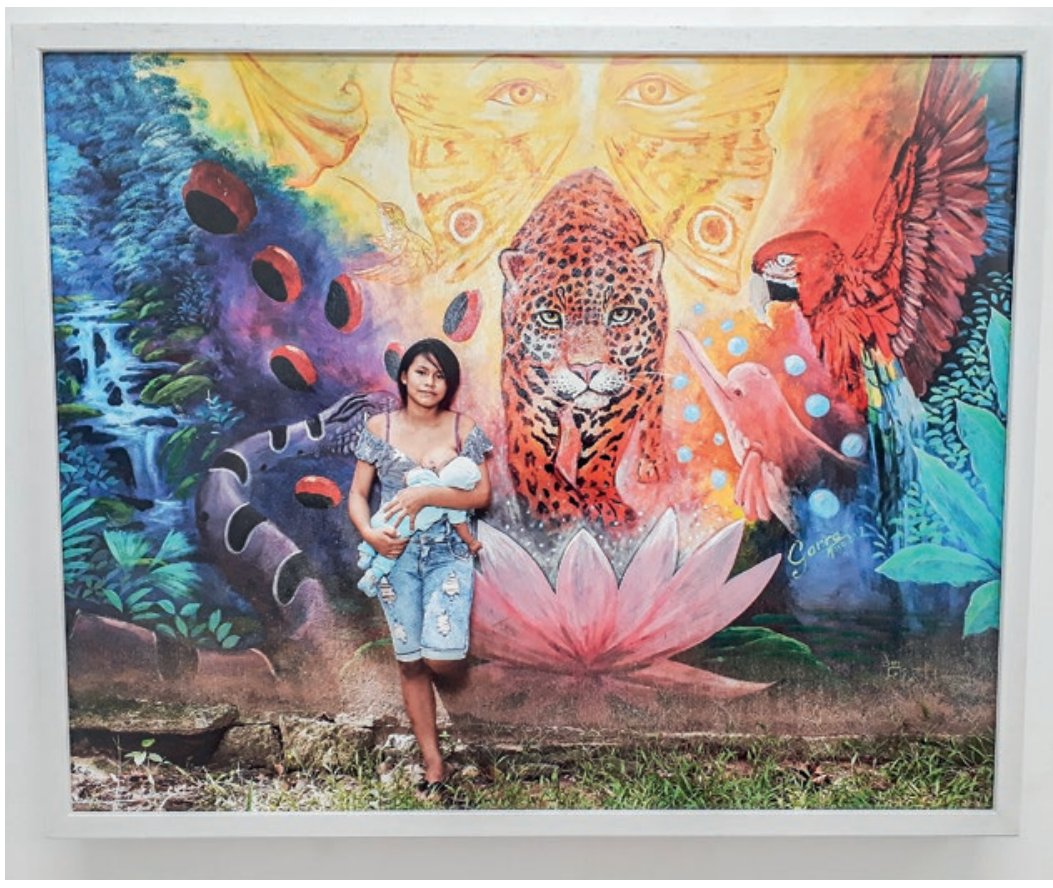

Une Amérindienne de 15 ans et son bébé de 1 mois. Elle ne prend pas de contraceptif.

du pays avec leurs impressionnantes tours de pilules, comprimés, pansements, gélules qu'ils vendent à la pièce. «Ces étalages de médicaments nous ont d'abord interpellés par leur «beauté», puis nous avons remar-

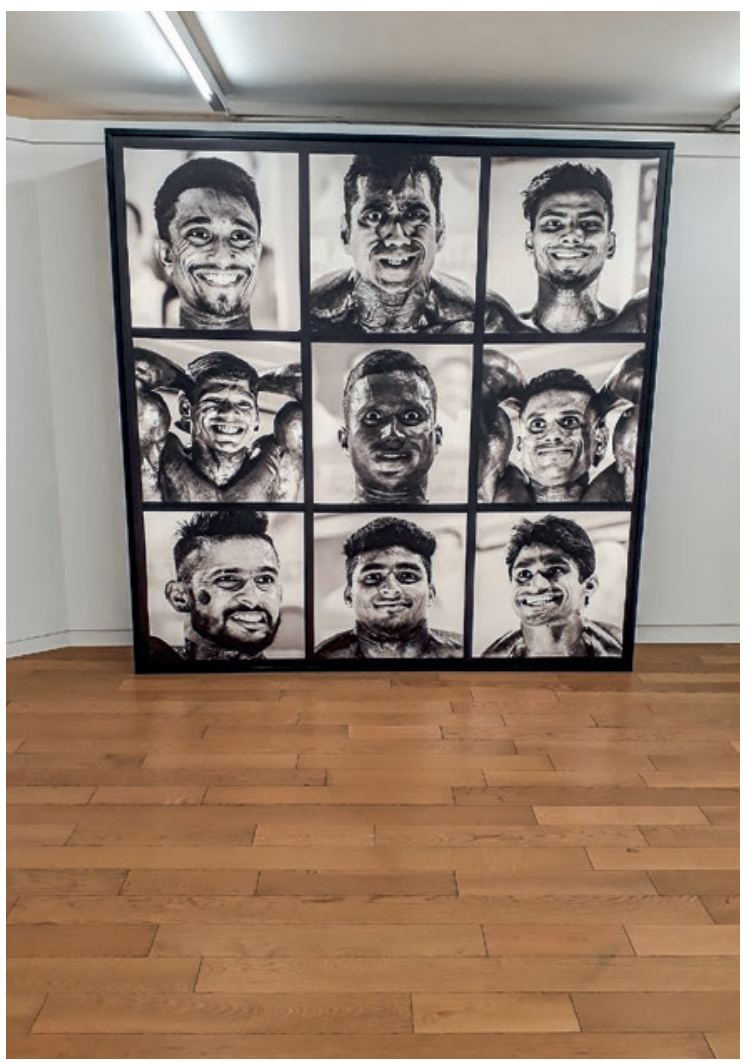

Les stéroïdes pour être musclés, mais quel effet sur la santé? qué que les substances vendues par ces marchands racontaient beaucoup d'Haïti: médicaments surdosés venant de Chine, médicaments abandonnés par les ONG. Mais surtout, nous étions frappés de voir le nombre de "patients» s'adressant à ces vendeurs, qui jouaient alors le rôle de prescripteurs», explique Arnaud Robert. $\mathrm{Au}$ fil de leurs recherches, notamment sur les mastodontes de l'industrie pharmaceutique Roche et Novartis, et des rencontres avec des consommatrices et consommateurs, ils se rendent compte qu'un médicament est plus qu'une simple notice d'utilisation. «Derrière un comprimé se cache la promesse de guérir, d'aller mieux, d'être plus fort, plus joyeux. Et ce, quasi immédiatement. On s'en remet à la pharma, car on est convaincu des promesses qu'elle fait miroiter. Mais on ne remet pas en question nos modes de vie ni notre consommation de médicaments", poursuit le journaliste.

Dans la première salle, des photos montrent des adolescentes et très jeunes femmes d'Amazonie péruvienne posant avec leurs enfants devant des murs aux graffitis colorés. Certaines ont eu leur premier enfant à 13 ans. A 20 ans, elles sont déjà mères trois fois. La solution la plus simple et efficace: les contraceptifs. Dans une autre pièce, une galerie de photos et posts Instagram illustrant le quotidien banal d'une jeune Américaine de 13 ans, collégienne et cheerleader. On lui a

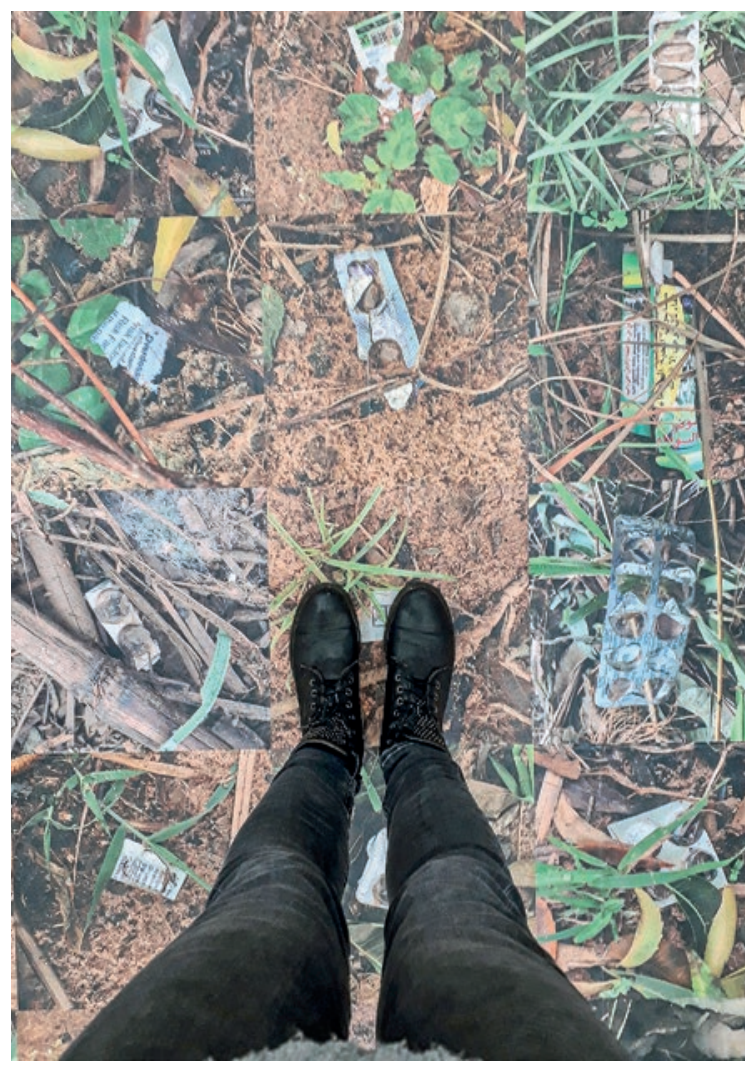

Sol jonché d'emballages de médicaments au Niger. 


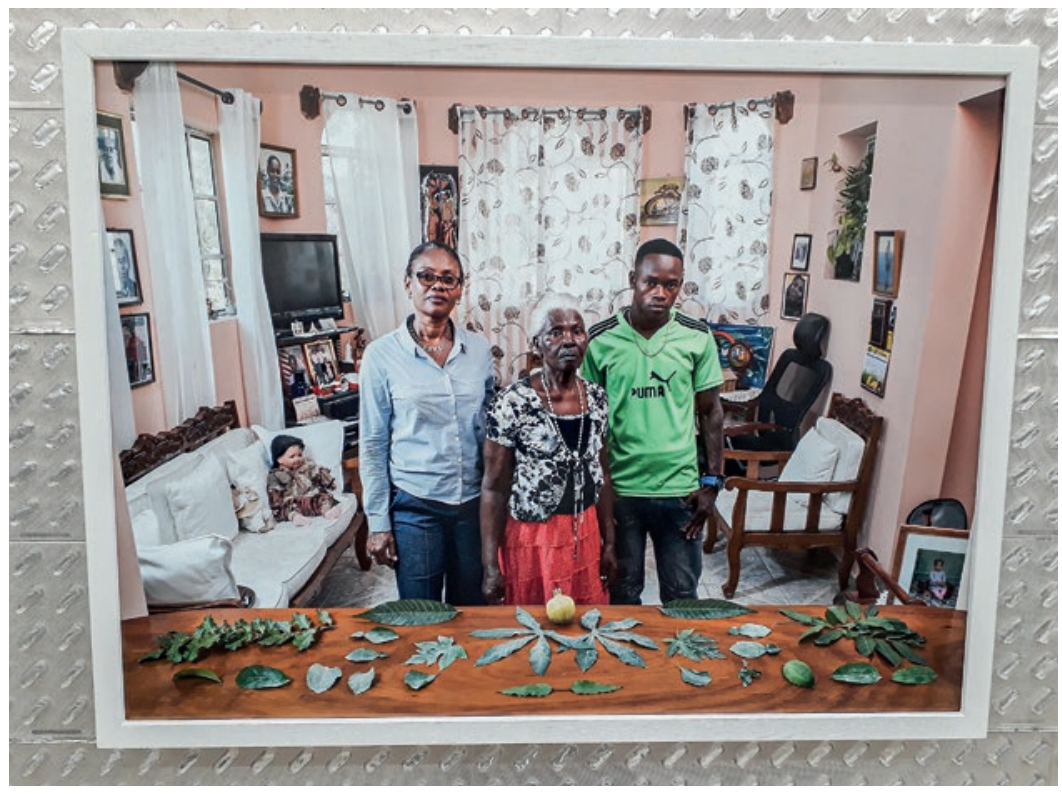

La pharmacie d'une famille haïtienne. Photo de la série Home Pharma.

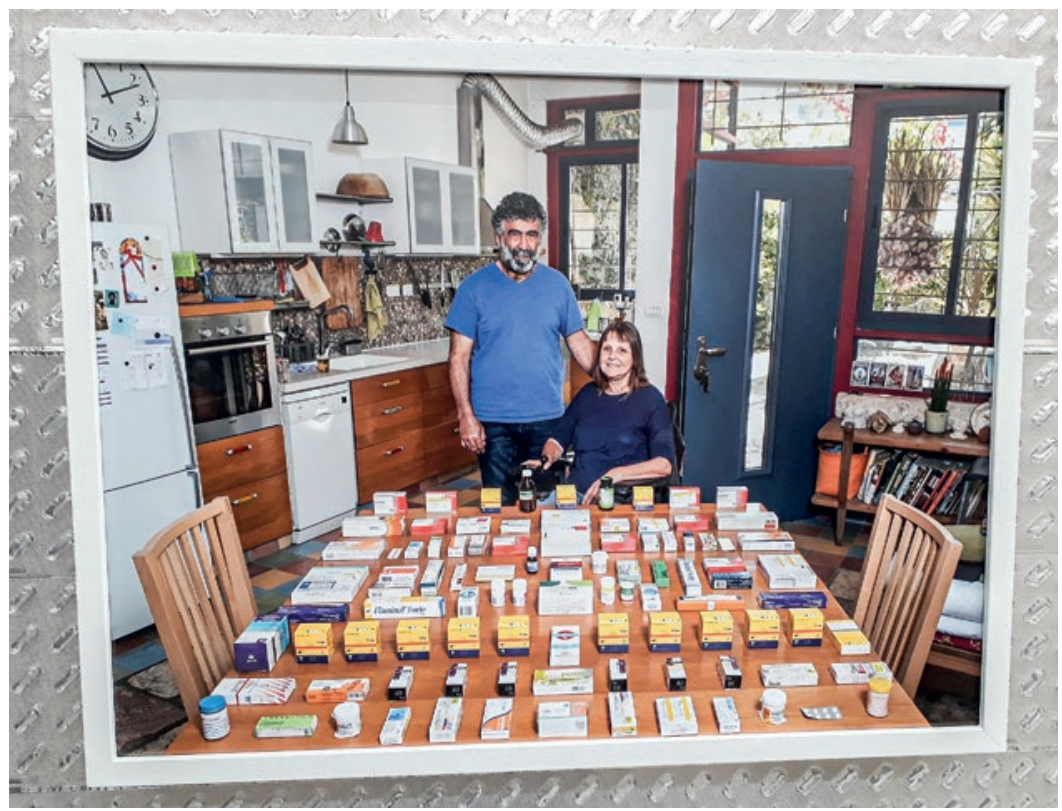

Un couple israélien et leur opulente réserve de médicaments. Photo de la série Home Pharma.

julia.rippstein[at]emh.ch

\section{HAPPY PILLS, un triptyque}

Issus de domaines différents mais reliés par la recherche documentaire et journalistique, Arnaud Robert et Paolo Woods étaient soucieux de proposer un projet multimédia. Outre l'exposition à La Ferme des Tilleuls, HAPPY PILLS est aussi un documentaire produit par Intermezzo Films, en coproduction avec RTS, SSR SRG et ARTE, ainsi qu'un livre, publié par delpire \& co, sorte de angle scientifique et philosophique. Plus d'informations: fermedestilleuls.ch/exhibition/happy-pills/ prolongement de l'exposition qui explore la question sous un diagnostiqué un trouble de l'attention qu'elle traite à l'Adderall. Apposée à côté des images, la citation d'un psychiatre et spécialiste des TDAH résume bien la problématique investiguée par HAPPY PILLS: «La médecine crée tout un tas de diagnostics qui définissent des troubles auxquels les médicaments donnent une réponse. La pilule marche rapidement, c'est ça que les gens aiment [...] Le problème, c'est que la solution vient toujours de l'extérieur. Notre bonheur vient de l'extérieur.»

\section{Porteurs d'espoir et révélateurs de l'intime}

Il y a aussi le gigolo italien qui prend un comprimé de Viagra avant chaque cliente pour se sentir performant et viril, ou le jeune Israélite gay qui avale une PrEP, pilule qui permet de prévenir une infection au VIH avant un rapport sexuel à risque. Une paroi entière composée de captures d'écran du site de rencontres gay Grindr semble illustrer le dilemme, que cette pilule révolutionnaire vient résoudre: succomber sans crainte à la luxure et rester "sain». Dans la même salle, des bodybuilders indiens aux muscles presque monstrueux et aux sourires forcés qui s'enfilent des stéroïdes comme on mangerait des tartines.

Un étage plus bas, la série de photographies Home Pharma montre des familles d'une trentaine de pays qui ont étalé tous les médicaments qu'elles possèdent à la maison: de l'opulence au strict nécessaire, des plantes médicinales aux antidépresseurs, la composition d'une pharmacie personnelle diffère d'un pays à l'autre, d'une culture à l'autre et révèle bien souvent le statut socio-économique. «Le dénominateur universel est la vision magique que l'on a de ces substances. La pandémie de COVID-19 a montré à quel point le monde entier projetait ses espoirs dans la pharma pour trouver un remède à cette crise», évoque Arnaud Robert.

Interpellante, l'exposition HAPPY PILLS incite le public à se positionner vis-à-vis de sa propre consommation de substances chimiques. Elle met le doigt sur la signification, parfois très intime et souvent inconsciente, du recours à un bien globalisé et ultrabanalisé. De quoi ne plus voir un comprimé de Dafalgan de la même manière...

Crédits photos Julia Rippstein 